\title{
Dietary chitosan improves nitrogen use and feed conversion in diets for mid- lactation dairy cows
}

\author{
Tiago A. Del Valle ${ }^{\mathrm{a}}$, Pablo G. de Paiva ${ }^{\mathrm{b}}$, Elmeson Ferreira de Jesus ${ }^{\mathrm{b}}$, Gustavo F. de Almeida ${ }^{\mathrm{a}}$, \\ Filipe Zanferari $^{\mathrm{a}}$, Artur G.B.V.B. Costa ${ }^{\mathrm{a}}$, Ives C.S. Bueno ${ }^{\mathrm{c}}$, Francisco P. Rennó ${ }^{\mathrm{a}, *, 1}$ \\ a Department of Animal Nutrition and Production, University of Sao Paulo, Pirassununga, Brazil \\ b Department of Animal Science, UNESP - Universidade Estadual Paulista "Júlio de Mesquita Filho", Jaboticabal, Brazil \\ ${ }^{c}$ Department of Animal Science, University of Sao Paulo, Pirassununga, Brazil
}

\section{A R T I C L E I N F O}

\section{Keywords:}

Chitin

Digestibility

Fatty acids

Milk yield

Ruminal fermentation

\begin{abstract}
A B S T R A C T
Feed additives and fat sources have been used to meet high productive dairy cow energy requirements. This study aimed to evaluate dietary chitosan and soybean oil effects on mid-lactation dairy cow intake, digestibility, metabolism and productive performance. Twenty-four Holstein cows (134.7 \pm 53.1 days in milk, $36.14 \pm 5.32 \mathrm{~kg} /$ day of milk yield, and $581.2 \pm 73.6 \mathrm{~kg}$ of body weight, Mean \pm SD) were used in a replicated $4 \times 4$ Latin square design with 21 -d periods, with $14 \mathrm{~d}$ of adaptation and $7 \mathrm{~d}$ for data collection. The treatment arrangement was a $2 \times 2$ factorial design with two levels of chitosan ( 0 and $4 \mathrm{~g} / \mathrm{kg}$ of dietary dry matter - DM) and two levels of soybean oil (0 and $33 \mathrm{~g} / \mathrm{kg}$ of dietary DM). Chitosan decreased intake only in diets without oil $(\mathrm{P}<0.05)$. Regardless of fat addition, chitosan increased DM and CP digestibility $(\mathrm{P}<0.05)$. Soybean oil and chitosan increased total serum cholesterol $(\mathrm{P}<0.05)$. Chitosan diet had higher urea plasma concentration than control diet $(\mathrm{CON})(\mathrm{P}<0.05)$. Over all, soybean oil increased propionate and decreased acetate ruminal molar proportion, and therefore decreased acetate:propionate ratio $(\mathrm{P}<0.05)$. Chitosan decreased milk yield, nitrogen use and feed conversion efficiencies in oil-diets $(\mathrm{P}<0.05)$. Soybean oil decreased short and medium milk fatty acids concentration $(\mathrm{P}<0.05)$. Chitosan had no effect on long-chain milk fatty acids in diets with soybean oil $(\mathrm{P}>0.05)$. However, in free oil-diets, chitosan increased milk polyunsaturated fatty acids concentration, nitrogen and energy efficiency. Chitosan addition in free-fat diets improved feed efficiency, increased milk unsaturated fatty acids concentration and association with soybean oil negatively affect animal performance.
\end{abstract}

\section{Introduction}

Energy requirements have been among the greatest challenges for ever-increasing lactation productivity dairy cows (Loften et al., 2014). One of the most studied strategies for this challenge is the dietary additives supplementation, to modulate ruminal digestion process (Van Nevel and Demeyer, 1988; Odongo et al., 2007; Silva et al., 2007). The most frequently used additives are primarily substances with antimicrobial activity, particularly ionophore, which has been successful in increasing the efficiency of protein and energy utilization (Van Nevel and Demeyer, 1988; Odongo et al., 2007; Silva et al., 2007). Antibiotic utilization in animal feeds, however, is facing reduced social acceptance because of the possible residues in animal products and the development of resistant strains of bacteria (Barton, 2000).

Goiri et al. (2009) proposed chitosan use as a modulator of ruminal fermentation and digestive processes. Chitosan is a nontoxic and biodegradable biopolymer that has been used on several applications in medicine and food preservation, primarily because of its antimicrobial activities. Chitosan is obtained by the deacetylation of chitin, the most abundant biopolymer in nature after cellulose, and an important component of the exoskeleton of insects and crustaceans (Kong et al., 2010). Goiri et al. (2010a) showed that chitosan inhibits in vitro biohydrogenation, and increases unsaturated fatty acids concentration in Rusitec ${ }^{\circ}$ assay while in their other study Goiri et al. (2010b) found that rumen propionate increased, ammonium decreased and no effect on intake and total tract digestibility in sheep.

Another strategy to meet energy requirements of high productive ruminants is to use feeds with high energy density, such as those rich in lipids. Lipids are known to increase the energy density of diets and may affect ruminal fermentation, altering milk fatty acids profile (Jenkins, 1993). Both chitosan and fat sources, alter ruminal fermentation. We hypothesized that chitosan and lipids association may improve ruminal

\footnotetext{
* Corresponding author.

E-mail address: francisco.renno@usp.br (F.P. Rennó).

${ }^{1}$ Bursar 1-C of National Council of Scientific and Technologic Development
} 
fermentation, productive performance, energy status and milk fatty acids profile. It is unknown if there are interaction effects between these two or if there are additive effects. This study evaluated the combined use of chitosan and soybean oil in dairy cow diets on ruminal fermentation, intake and digestibility, $\mathrm{N}$ balance, productive performance and milk fatty acids profile.

\section{Material and methods}

The Ethics Committee of the School of Veterinary Medicine and Animal Science of the University of São Paulo, under number 3057/ 2013, approved the experimental procedures.

\subsection{Animals, experimental design and diets}

Twenty-four Holstein (eight ruminal cannulated) with $134.7 \pm 53.1$ days in milk, $581.2 \pm 73.6 \mathrm{~kg}$ of body weight and $36.14 \pm 5.32 \mathrm{~kg} / \mathrm{d}$ of milk yield (mean $\pm \mathrm{SD}$ ), were housed in individual pens, with $17.5 \mathrm{~m}^{2}$ of area, on sand beds and forced ventilation. Animals were divided into six groups, according to ruminal cannula presence, days in milk and milk yield. Animals within groups were randomly allocated into a treatments sequence, in a Latin Square design. Experimental diets were formulated according NRC (2001) recommendations, as follows (Table 1): CON: control diet with no chitosan and no soybean oil; CHI: chitosan diet, with chitosan ( $4 \mathrm{~g} / \mathrm{kg}$ of dry matter) and without soybean oil; SO: soybean oil diet, with no chitosan and with soybean oil ( $33 \mathrm{~g} / \mathrm{kg}$ of dry matter); and CSO: chitosan and soybean oil, diet with chitosan and with soybean oil. The Chitosan was manufactured from Polymar (Polymar Science and Nutrition, Fortaleza, Brazil) and dosage

Table 1

Ingredients and chemical composition of experimental diets containing chitosan and soybean oil.

\begin{tabular}{|c|c|c|c|c|}
\hline \multirow[t]{2}{*}{ Item } & \multicolumn{4}{|c|}{ Experimental diets ${ }^{\mathrm{a}}$} \\
\hline & CON & $\mathrm{CHI}$ & So & $\mathrm{CSO}$ \\
\hline \multicolumn{5}{|l|}{ Ingredients (g/kg DM) } \\
\hline Corn silage & 500 & 500 & 500 & 500 \\
\hline Ground corn & 282 & 279 & 242 & 240 \\
\hline Soybean meal & 191 & 189 & 198 & 196 \\
\hline Soybean oil & - & - & 33.0 & 32.7 \\
\hline Chitosan & - & 4.0 & - & 4.0 \\
\hline Sodium bicarbonate & 8.0 & 7.9 & 8.0 & 7.9 \\
\hline Limestone & 7.0 & 6.9 & 7.0 & 6.9 \\
\hline Urea & 3.2 & 3.2 & 3.2 & 3.2 \\
\hline Dicalcium phosphate & 3.0 & 3.0 & 3.0 & 3.0 \\
\hline Salt & 3.0 & 3.0 & 3.0 & 3.0 \\
\hline Mineral mixture ${ }^{\mathrm{b}}$ & 2.0 & 2.0 & 2.0 & 2.0 \\
\hline Ammonium Sulphate & 1.0 & 1.0 & 1.0 & 1.0 \\
\hline \multicolumn{5}{|l|}{ Chemical composition, g/kg DM } \\
\hline Dry matter ${ }^{c}$ & 529 & 529 & 531 & 531 \\
\hline Non-fiber carbohydrates ${ }^{\mathrm{d}}$ & 393 & 393 & 367 & 367 \\
\hline Neutral-detergent fiber (NDF) & 349 & 348 & 344 & 343 \\
\hline Acid-detergent fiber & 189 & 189 & 188 & 188 \\
\hline Crude Protein & 172 & 173 & 171 & 172 \\
\hline Ash & 60.8 & 60.7 & 60.4 & 60.3 \\
\hline Acid-detergent lignin & 34.3 & 34.2 & 33.6 & 33.6 \\
\hline Ether Extract & 31.1 & 30.9 & 64.1 & 63.7 \\
\hline Net energy $3 \mathrm{x}^{\mathrm{e}}$ & 6.94 & 6.94 & 7.32 & 7.28 \\
\hline
\end{tabular}

${ }^{\text {a }}$ CON: Control diet; CHI: Chitosan diet; SO: Soybean oil diet; and CSO: Chitosan and soybean oil diet.

${ }^{\mathrm{b}}$ Each kg contains: $205 \mathrm{~g}$ of Ca; $60 \mathrm{~g}$ of P; $35 \mathrm{~g}$ of K; $70 \mathrm{~g}$ of Na; $20 \mathrm{~g}$ of S; $20 \mathrm{~g}$ of Mg; $2500 \mathrm{mg}$ of $\mathrm{Zn} ; 1600 \mathrm{mg}$ of $\mathrm{Mn} ; 700 \mathrm{mg}$ of Cu; $700 \mathrm{mg}$ of Fe; $40 \mathrm{mg}$ of I; $19 \mathrm{mg}$ of Se; $10 \mathrm{mg}$ of Cr; 200,000 IU of Vitamin A, 50,000 IU of Vitamin D, and $1500 \mathrm{IU}$ of Vitamin E.

c $\mathrm{g} / \mathrm{kg}$ of natural matter.

d Non-fiber carbohydrate $(\mathrm{g} / \mathrm{kg})=1000-[$ (crude protein $(\mathrm{g} / \mathrm{kg})-$ crude protein from urea $(\mathrm{g} / \mathrm{kg})+$ urea $(\mathrm{g} / \mathrm{kg}))+\mathrm{NDF}(\mathrm{g} / \mathrm{kg})+$ ether extract $(\mathrm{g} / \mathrm{kg})+\mathrm{ash}(\mathrm{g} / \mathrm{kg})]$ in which values were expressed in percentage, from Hall (2000).

e Calculated according to NRC (2001): $\mathrm{NE}_{\mathrm{L3x}}(\mathrm{MJ} / \mathrm{kg} \mathrm{DM})=\left[0.245^{*} \mathrm{TDN}_{1 \mathrm{x}}(\mathrm{g} / \mathrm{kg})\right.$ $-0.12] * 4.184 \mathrm{MJ} / \mathrm{Mcal}$, were $\mathrm{TDN}_{1 \mathrm{x}}(\mathrm{g} / \mathrm{kg})=\mathrm{tdNFC}+\mathrm{tdCP}+(\mathrm{tdFA} * 2.25)+\mathrm{tdNDF}$. was determinated to provide almost $150 \mathrm{mg} / \mathrm{kg}$ body weight intake, according to Araújo et al. (2015) and Mingotti et al. (2016). Chitosan (average MW 500,000 g) had a pH of 8.81 and a deacetylation degree of $86.6 \%$. Soybean oil level was added to the diet to provide $64 \mathrm{~g} / \mathrm{kg}$ of ether extract in the diet dry matter (NRC, 2001). Corn silage, ground corn and soybean meal were the main ingredients of the diets. Chitosan and soybean oil were added to the concentrate and offered as total mixed ration.

\subsection{Data and sample collection}

Each experimental period lasted for 21 days, with 14 days for adaptation and seven days for sampling. Cows were fed twice daily, at 7:00 a.m. and 1:00 p.m., with the quantity determined according to the amount of refusal from the previous day to maintain a percentage of refusal between $5 \%$ and $10 \%$ of supplied diet. Samples of corn silage and refusals were daily collected throughout the sampling period, stored frozen, and pooled in a composite sample for subsequent chemical analyses. Concentrates were collected at the animal's food factory, once for each week of sampling.

On days 16-18 of each experimental period, fecal samples were taken from each cow, after a.m. and p.m. daily milking. Samples of feeds, refusals and feces were dried at $60{ }^{\circ} \mathrm{C}$ in a forced-air oven for $72 \mathrm{~h}$ and processed in a Wiley mill either through a $1 \mathrm{~mm}$ or a $2 \mathrm{~mm}$ sieve. Samples of feeds, refusals and feces, ground at 1-mm, were analyzed for dry matter (DM, method 930.15; AOAC, 2000), crude protein (CP, $\mathrm{N} \times 6.25$; Kjeldahl method -984.13; AOAC, 2000), ether extract (EE, method 920.39; AOAC, 2000), acid-detergent fiber and lignin (ADF and Lignin, method 973.18; AOAC, 2000), ash (method 942.05; AOAC, 2000) and neutral-detergent fiber (NDF) using $\alpha$-amylase and without addition of sodium sulfite (Van Soest et al., 1991).

Indigestible acid-detergent fiber (iADF) was used as internal marker to estimate fecal excretion and apparent digestibility of nutrients. Samples of feeds, refusals and feces, ground at 2-mm, were placed in bags of nonwoven textile $\left(100 \mathrm{~g} / \mathrm{m}^{2}\right.$ of weight per area unit) and were incubated for $288 \mathrm{~h}$ in the rumen of two Holstein cows (Casali et al., 2008), previously adapted to a high-concentrate diet. After removal, the bags were washed in running tap water, dried at $60{ }^{\circ} \mathrm{C}$ in a forcedair oven and digested by an acid-detergent solution in an fiber analyzer (TE149 ${ }^{\circ}$, Tecnal Equipamentos Científicos, Piracicaba, Brazil) to obtain the indigestible acid-detergent fiber (method 973.18; AOAC, 2000). Apparent digestibility coefficient were estimated through the intake and fecal excretion data.

Ruminal liquid was collected on day 20 of each experimental period at zero, two, four, six, eight, ten and $12 \mathrm{~h}$ after morning feeding from four rumen cannulated cows. Rumen $\mathrm{pH}$ value was recorded immediately after collection with a digital pH meter (MB-10 ${ }^{\circ}$, Marte Científica, Santa Rita do Sapucaí, Brazil). The concentrations of volatile fatty acids (VFA) in the rumen fluid were measured by gas chromatography as described by Shen et al. (2004), and the $\mathrm{N}-\mathrm{NH}_{3}$ content was analyzed using phenol-hypochlorite (Broderick and Kang, 1980).

Blood samples were collected on the 15th day of each experimental period, before the morning feeding, by coccygeal vein or artery puncture. Samples were centrifuged at $800 \times g$ for $10 \mathrm{~min}$, and the serum was collected and frozen. The analyses were performed with commercially available colorimetric kits (glucose: cat. no. K-082; total cholesterol: cat. no. K-083; high-density lipoprotein - HDL cholesterol: cat. no. K-015; urea: cat. no. K-056; aspartate aminotransferase - AST: cat. no. K-048; and gamma-glutamyl transpeptidase - GGT: cat. no. K080; Bioclin ", Belo Horizonte, Brazil). Readings were determined with a semi-automatic spectrophotometer (SBA $200^{\circ}$, CELM, São Caetano do Sul, Brazil).

Daily urine volume was estimated from creatinine concentration $(\mathrm{mg} / \mathrm{L})$ in spot samples obtained on the 16th and 17th day of each experimental period, four hours after morning feeding. Creatinine concentrations were analyzed with a biochemical colorimetric kit 
Table 2

Intake and digestibility of dry matter and nutrients in lactating dairy cows fed diets containing chitosan and soybean oil.

\begin{tabular}{|c|c|c|c|c|c|c|c|c|}
\hline \multirow[t]{2}{*}{ Item } & \multicolumn{4}{|l|}{ Diets $^{1}$} & \multirow[t]{2}{*}{ SEM $^{2}$} & \multicolumn{3}{|l|}{$P$-value } \\
\hline & $\mathrm{CON}$ & $\mathrm{CHI}$ & so & $\mathrm{CSO}$ & & Chitosan & Soy. oil ${ }^{3}$ & $\mathrm{CHI} \times \mathrm{SO}^{4}$ \\
\hline \multicolumn{9}{|l|}{ Intake, $\mathrm{kg}$ /day } \\
\hline Dry matter & $22.5^{\mathrm{a}}$ & $21.2^{\mathrm{b}}$ & $20.0^{\mathrm{c}}$ & $20.1^{\mathrm{c}}$ & 0.40 & 0.020 & $<0.001$ & 0.009 \\
\hline $\mathrm{NDF}^{5}$ & $7.82^{\mathrm{a}}$ & $7.32^{\mathrm{b}}$ & $6.79^{c}$ & $6.83^{c}$ & 0.15 & 0.027 & $<0.001$ & 0.010 \\
\hline Crude protein & $3.90^{\mathrm{a}}$ & $3.69^{\mathrm{b}}$ & $3.40^{c}$ & $3.45^{\mathrm{c}}$ & 0.07 & 0.093 & $<0.001$ & 0.009 \\
\hline Ether extract & 0.70 & 0.67 & 1.29 & 1.29 & 0.04 & 0.426 & $<0.001$ & 0.489 \\
\hline \multicolumn{9}{|c|}{ Total tract apparent digestibility, $\mathrm{g} / \mathrm{kg}$} \\
\hline Dry matter & 725 & 740 & 727 & 750 & 3.7 & 0.003 & 0.326 & 0.558 \\
\hline $\mathrm{NDF}^{5}$ & 601 & 599 & 584 & 603 & 5.3 & 0.341 & 0.479 & 0.253 \\
\hline Crude protein & 742 & 767 & 754 & 782 & 4.2 & $<0.001$ & 0.055 & 0.881 \\
\hline Ether extract & 851 & 841 & 891 & 898 & 7.2 & 0.889 & $<0.001$ & 0.443 \\
\hline
\end{tabular}

${ }^{1}$ CON: Control diet; CHI: Chitosan diet; SO: Soybean oil diet; and CSO: Chitosan and soybean oil diet.

${ }^{2}$ SEM: Standard error of the mean.

${ }^{3}$ Soybean oil effect.

${ }^{4}$ Chitosan $\times$ Soybean oil interaction.

${ }^{5}$ NDF: Neutral-detergent fiber.

a,b,c Values within a row with different superscripts differ significantly at $P<0.05$.

(kinetic creatinine: cat. no. K-067, Bioclin) in a semi-automatic spectrophotometer (SBA 200), and a daily creatinine excretion rate of $24.05 \mathrm{mg} / \mathrm{kg}$ of body weight was assumed (Chizzotti et al., 2008). The total excretion of uric acid (uric acid stable liquid: cat. no. K-052, Bioclin; determined in a semi-automatic spectrophotometer) and allantoin (Fujihara and Yamaguchi, 1978) in urine and milk were considered to be the total excretion of purine derivatives. Microbial protein synthesis was estimated from these concentrations considering molar excretion of allantoin (urine and milk) and uric acid (urine). We considered 0.116 of ratio between $\mathrm{N}$ purine and microbial $\mathrm{N}$, and 0.83 of intestinal microbial purines digestibility (Chen and Gomes, 1992). According to González-Ronquillo et al. (2003), urinary recovery of absorbed purines was assumed to be 0.70 and endogenous contribution was estimated as $0.512 \mathrm{mmol} / \mathrm{kg}^{0.75}$ BW. Energy intake was estimated according NRC (2001) equations. Nitrogen efficiency of use was assessed by $\mathrm{N}$ milk yield and $\mathrm{N}$ intake ratio (Arndt et al., 2015).

Cows were mechanically milked twice daily, at 6:00 a.m. and 4:00 p.m., and the milk yield was weighed daily for the last seven days of each experimental period. From 16th to 18th days of each period, milk samples of two daily milking, were collected for composition analyses. Fresh samples were analyzed for crude protein, fat and lactose, using ultrasonic milk analyzer MCC (Milcotronic Company, Nova Zagora, 8900, Bulgaria). Milk yields were corrected for $3.5 \%$ fat content, according to Sklan et al. (1994).

Milk fatty acid profiles were evaluated in the composite sample obtained on the 16th day of each experimental period. Fatty acid extraction was performed according to Feng et al. (2004) and methylated according to Kramer et al. (1997). Readings were performed in gas chromatograph (GC Shimatzu model GC-2010, Kyoto, Japan), using a capillary column (Sigma Aldrich Supelco SP- $2560^{\circ}$, St. Louis, MO, USA) and hydrogen $\left(\mathrm{H}_{2}\right)$ as the carrier gas. Standards $\mathrm{C} 4$ to $\mathrm{C} 24$ fatty acids Sigma Aldrich Supelco TM37 ${ }^{\circ}$, St. Louis, MO, USA; vaccenic acid (C18:1 trans-11) (Sigma Aldrich V038-1 G ${ }^{\circ}$, St. Louis, MO, USA), C18:2 trans-10, cis-12 (NU-Chek-Prep UC-61M $100 \mathrm{mg}^{\circ}$, Elysian, MN, USA) and C18:2 cis-9, trans-11 (NU-Chek-Prep UC-60M $100 \mathrm{mg}$, Elysian, MN, USA) were used. Internal standards of C19:0 was used to correct methylation losses.

\subsection{Statistical analyses}

Data were analyzed using PROC MIXED (Statistical Analysis System for Windows, version 9.0, SAS Institute Inc., Cary, NC, USA, 2003), according to the following statistical model:
$Y_{i j k l m}=\mu+C_{i}+S O_{j}+C^{*} S O_{i j}+P_{k}+S_{l}+a_{m}\left(s_{l}\right)+e_{i j k l m}$

where: $Y_{i j k l m}$ was the observed value in the animal $\mathrm{m}$, from 1 square, in the kth period, which received the lth level of chitosan and the $\mathrm{j}^{\text {th }}$ level of soybean oil; $\mu$ : overall mean; $C_{i}$ was the fixed effect of chitosan; $S O_{j}$ was the fixed effect of soybean oil; $C^{*} S O_{i j}$ was the chitosan and soybean oil interaction effect; $P_{k}$ was the fixed effect of experimental period; $S_{l}$ was the fixed effect of the Latin square; $a_{m}\left(s_{\nu}\right)$ was the random effect of animal within square and $e_{i j k l m}$ was the random residual error. Degrees of freedom were corrected by Kenward and Roger (1997) methodology. Means were adjusted by LSMEANS option and when there was chitosan*soybean oil interaction effect, means were studied by the PDIFF means test.

The ruminal fermentation variables $\left(\mathrm{pH}, \mathrm{N}-\mathrm{NH}_{3}\right.$, acetate, propionate, butyrate and total VFA) were analyzed as repeated measures in PROC MIXED of SAS 9.0, considering in the statistical model the effects of animal, period, chitosan, and soybean oil and the effects of time with its interactions with the other effects above mentioned effects. Akaike methodology was used to choose the best covariance structure: TOEP was used for $\mathrm{pH}$, total VFA concentration and propionate proportion; $\mathrm{ARH}(1)$ for N-NH3; AR(1) for acetate proportion and acetate to propionate ratio and CS for propionate proportion. Differences were considered significant at the 0.05 level.

\section{Results}

\subsection{Intake and digestibility}

A significant soybean oil $\times$ chitosan interaction effect was observed for DM, CP and NDF intakes ( $\mathrm{P} \leq 0.010$; Table 2). Chitosan supplementation to low fat diets decreased feed intake $(P \leq 0.05)$, while chitosan had no effect on dry matter intake in fat supplemented diets $(P>0.05)$. Chitosan showed no effect on EE intake and digestibility coefficients $(P \geq 0.426)$. Soybean oil increased $E E$ intake and digestibility $(\mathrm{P}<0.001)$ and chitosan increased DM and $\mathrm{CP}$ digestibility $(\mathrm{P} \leq 0.003)$.

\subsection{Ruminal fermentation}

There was an expected physiological time effect on evaluated ruminal variables $(\mathrm{P} \leq 0.005$; Table 3 and Fig. 1$)$. Chitosan had no effect on ruminal fermentation variables, regardless fat addition $(P \geq 0.111)$. Soybean oil decreased ruminal acetate concentration and acetate to propionate ratio and increased ruminal propionate proportion $(\mathrm{P} \leq 0.004)$. 
Table 3

Ruminal fermentation parameters of lactating dairy cows fed diets containing chitosan and soybean oil.

\begin{tabular}{|c|c|c|c|c|c|c|c|c|c|}
\hline \multirow[t]{2}{*}{ Item } & \multicolumn{4}{|l|}{$\operatorname{Diets}^{\mathrm{a}}$} & \multirow[t]{2}{*}{ SEM $^{\mathrm{b}}$} & \multirow[b]{2}{*}{ Time } & \multicolumn{3}{|l|}{ P-value } \\
\hline & $\mathrm{CON}$ & $\mathrm{CHI}$ & SO & $\mathrm{CSO}$ & & & Chitosan & Soybean oil & Int. $\mathrm{CHI} \times \mathrm{SO}^{\mathrm{c}}$ \\
\hline $\mathrm{pH}$ & 6.34 & 6.46 & 6.43 & 6.48 & 0.048 & 0.001 & 0.353 & 0.580 & 0.632 \\
\hline $\mathrm{N}-\mathrm{NH}_{3}, \mathrm{~g} / \mathrm{L}$ & 0.339 & 0.336 & 0.281 & 0.317 & 0.012 & $<0.001$ & 0.386 & 0.244 & 0.443 \\
\hline $\mathrm{VFA}^{\mathrm{d}}, \mathrm{mMol} / \mathrm{L}$ & 129 & 123 & 120 & 115 & 2.54 & $<0.001$ & 0.111 & 0.061 & 0.383 \\
\hline \multicolumn{10}{|c|}{ Molar proportion } \\
\hline Acetate & 0.660 & 0.661 & 0.619 & 0.641 & 0.004 & $<0.001$ & 0.136 & 0.004 & 0.235 \\
\hline Propionate & 0.211 & 0.215 & 0.248 & 0.240 & 0.003 & 0.005 & 0.564 & 0.001 & 0.545 \\
\hline Butyrate & 0.129 & 0.124 & 0.132 & 0.119 & 0.002 & $<0.001$ & 0.201 & 0.897 & 0.522 \\
\hline $\mathrm{A}: \mathrm{P}^{\mathrm{e}}$ & 3.17 & 3.13 & 2.56 & 2.74 & 0.053 & $<0.001$ & 0.395 & 0.001 & 0.365 \\
\hline
\end{tabular}

a CON: Control diet; CHI: Chitosan diet; SO: Soybean oil diet; and CSO: Chitosan and soybean oil diet.

b SEM: Standard error of the mean.

c Soybean oil $\times$ chitosan interaction effect.

d VFA: total volatile fatty acids.

${ }^{\mathrm{e}}$ A:P: Acetate to propionate ruminal molar ratio.

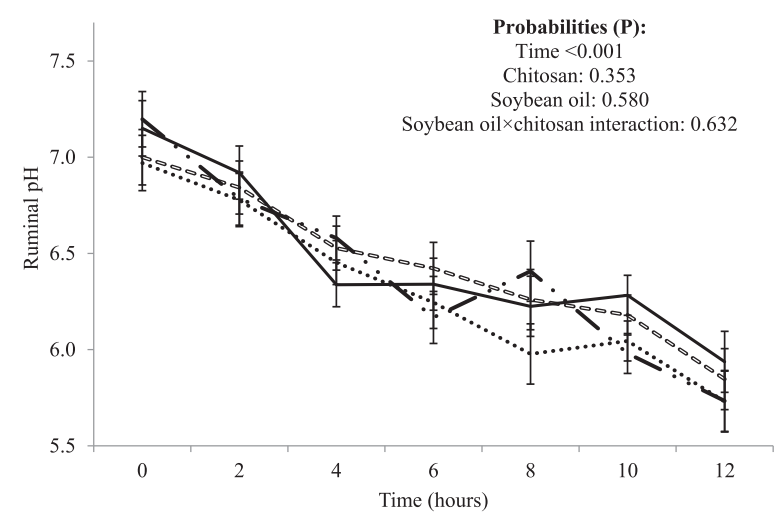

Fig. 1. Ruminal $\mathrm{pH}$ of dairy cows fed diets containing chitosan and soybean oil. Control

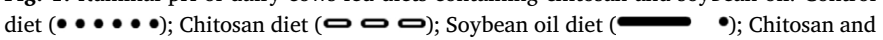
soybean oil diet (ـ) hours of feeds $(\uparrow)$; Means ( \pm SEM).

\subsection{Blood metabolism}

Experimental diets had no effect on glucose serum concentrations and GGT activity $(P \leq 0.191$; Table 4$)$. There was soybean oil $\times$ chitosan interaction effect on serum urea concentration $(P<0.001)$. Chitosan supplementation to low fat diets increased serum urea $(\mathrm{P} \leq 0.05)$, while chitosan had no effect on serum urea on those animals fed with fat supplemented diets $(\mathrm{P}>0.05)$. Regardless fat supplementation, chitosan increased serum total cholesterol $(P=0.012)$. Furthermore, dietary fat addition increased HDL-cholesterol, total cholesterol, and AST activity $(\mathrm{P} \leq 0.010)$.

\subsection{Milk yield and composition}

There was a soybeans oil $\times$ chitosan interaction effect on milk, protein and lactose production and feed conversion efficiency $(\mathrm{P} \leq 0.033$; Table 5). Chitosan increased feed conversion efficiency $(P \leq 0.05)$ and had no effect on milk, protein and lactose production of animals fed diets without soybean oil $(P>0.05)$, while chitosan decreased milk yield and feed conversion efficiency of those animals fed fat supplemented diets $(\mathrm{P} \leq 0.05)$. Soybean oil decreased fat-corrected milk and milk fat content, and increased lactose milk content $(\mathrm{P} \leq 0.05)$. Chitosan in diets without oil had not affect on milk composition $(\mathrm{P}>0.05)$.

\subsection{Energy and $N$ usage and microbial protein synthesis}

A significant soybean oil $\times$ chitosan interaction effect was observed on net energy intake and efficiency of energy usage $(P \leq 0.014$; Table 6). Animals fed diets without soybean oil showed decreased net energy intake and increased energy usage efficiency when chitosan was dietary added $(\mathrm{P} \leq 0.05)$. However, chitosan decreased energy usage efficiency $(P \leq 0.05)$ and had no effect on net energy intake of those animals fed fat supplemented diets $(P>0.05)$. Regardless chitosan addition, soybean oil supplementation decreased milk net energy $(\mathrm{P}<0.001)$.

There was a significant soybean oil $\times$ chitosan interaction effect on $\mathrm{N}$ intake, $\mathrm{N}$ milk secretion and $\mathrm{N}$ usage efficiency $(\mathrm{P} \leq 0.047)$. In

Table 4

Serum parameters of lactating Holstein cows fed diets containing chitosan and soybean oil.

\begin{tabular}{|c|c|c|c|c|c|c|c|c|}
\hline \multirow[t]{2}{*}{ Item } & \multicolumn{4}{|l|}{ Diets $^{1}$} & \multirow[t]{2}{*}{$\mathrm{SEM}^{2}$} & \multicolumn{3}{|l|}{ P-value } \\
\hline & $\mathrm{CON}$ & $\mathrm{CHI}$ & SO & $\mathrm{CSO}$ & & Chitosan & Soybean oil & $\mathrm{CHI} \times \mathrm{SO}^{3}$ \\
\hline Glucose, g/L & 0.674 & 0.676 & 0.689 & 0.647 & 0.015 & 0.497 & 0.797 & 0.441 \\
\hline Total Cholesterol, g/L & 1.469 & 1.700 & 2.143 & 2.249 & 0.078 & 0.012 & $<0.001$ & 0.341 \\
\hline HDL Cholesterol $^{4}, \mathrm{~g} / \mathrm{L}$ & 0.574 & 0.577 & 0.636 & 0.652 & 0.016 & 0.679 & 0.003 & 0.767 \\
\hline Urea, g/L & $0.329^{c}$ & $0.418^{\mathrm{a}}$ & $0.381^{\mathrm{a}, \mathrm{b}}$ & $0.359^{\mathrm{b}, \mathrm{c}}$ & 0.009 & 0.004 & 0.734 & $<0.001$ \\
\hline $\mathrm{AST}^{5}, \mathrm{IU} / \mathrm{L}$ & 59.06 & 57.20 & 66.95 & 64.89 & 1.959 & 0.506 & 0.010 & 0.972 \\
\hline $\mathrm{GGT}^{6}, \mathrm{IU} / \mathrm{L}$ & 35.90 & 37.70 & 37.31 & 38.69 & 1.003 & 0.191 & 0.320 & 0.863 \\
\hline
\end{tabular}

\footnotetext{
${ }^{1}$ CON: Control diet; CHI: Chitosan diet; SO: Soybean oil diet; and CSO: Chitosan and soybean oil diet.

2 SEM: Standard error of the mean.

${ }^{3}$ Soybean oil $\times$ chitosan interaction effect.

${ }^{4}$ HDL Cholesterol: high-density lipoprotein.

5 AST: Aspartate aminotransferase.

${ }^{6}$ GGT: Gamma-glutamyl transferase.

a,b,c Values within a row with different superscripts differ significantly at $P<0.05$.
} 
Table 5

Milk yield and composition of lactating dairy cows fed diets containing chitosan and soybean oil.

\begin{tabular}{|c|c|c|c|c|c|c|c|c|}
\hline \multirow[t]{2}{*}{ Item } & \multicolumn{4}{|l|}{ Diets $^{1}$} & \multirow[t]{2}{*}{$\mathrm{SEM}^{2}$} & \multicolumn{3}{|l|}{$\mathrm{P}$-value } \\
\hline & $\mathrm{CON}$ & $\mathrm{CHI}$ & so & $\mathrm{CSO}$ & & Chitosan & Soybean Oil & $\mathrm{CHI} \times \mathrm{SO}^{3}$ \\
\hline \multicolumn{9}{|c|}{ Production, $\mathrm{kg} / \mathrm{d}$} \\
\hline Milk Yield & $32.8^{\mathrm{a}}$ & $33.6^{\mathrm{a}}$ & $32.9^{\mathrm{a}}$ & $31.3^{\mathrm{b}}$ & 0.548 & 0.386 & 0.024 & 0.023 \\
\hline $\mathrm{FCM}^{4}$ & 34.5 & 34.7 & 30.9 & 30.4 & 0.636 & 0.866 & $<0.001$ & 0.606 \\
\hline Fat & 1.25 & 1.24 & 1.03 & 1.04 & 0.027 & 0.936 & $<0.001$ & 0.789 \\
\hline Protein & $1.00^{\mathrm{a}}$ & $1.02^{\mathrm{a}}$ & $1.01^{\mathrm{a}}$ & $0.96^{\mathrm{b}}$ & 0.018 & 0.228 & 0.099 & 0.030 \\
\hline Lactose & $1.50^{\mathrm{a}}$ & $1.53^{\mathrm{a}}$ & $1.52^{\mathrm{a}}$ & $1.43^{\mathrm{b}}$ & 0.027 & 0.270 & 0.089 & 0.033 \\
\hline $\mathrm{FCE}^{5}, \mathrm{~kg} / \mathrm{kg}$ & $1.48^{\mathrm{c}}$ & $1.62^{\mathrm{a}, \mathrm{b}}$ & $1.68^{\mathrm{a}}$ & $1.59^{\mathrm{b}}$ & 0.031 & 0.365 & 0.002 & $<0.001$ \\
\hline \multicolumn{9}{|c|}{ Milk concentration, $\mathrm{g} / \mathrm{kg}$} \\
\hline Fat & 38.0 & 37.2 & 31.7 & 33.5 & 0.773 & 0.607 & $<0.001$ & 0.198 \\
\hline Protein & 30.5 & 30.4 & 30.9 & 30.7 & 0.154 & 0.316 & 0.092 & 0.872 \\
\hline Lactose & 45.6 & 45.4 & 46.2 & 46.1 & 0.202 & 0.393 & $<0.001$ & 0.765 \\
\hline
\end{tabular}

${ }^{1}$ CON: Control diet; CHI: Chitosan diet; SO: Soybean oil diet; and CSO: Chitosan and soybean oil diet.

${ }^{2}$ SEM: Standard error of the mean.

${ }^{3}$ Chitosan $\times$ Soybean oil interaction effect.

${ }^{4}$ FCM: $3.5 \%$ fat corrected milk.

${ }^{5}$ FCE: feed conversion efficiency (milk yield: dry matter intake ratio).

a,b,c Values within a row with different superscripts differ significantly at $\mathrm{P}<0.05$.

animals fed fat supplemented diets, chitosan addition had no effect on $\mathrm{N}$ intake $(\mathrm{P}>0.05)$, decreased milk $\mathrm{N}$ secretion $(\mathrm{P} \leq 0.05)$ and, therefore, decreased $\mathrm{N}$ usage efficiency $(\mathrm{P} \leq 0.05)$. Although, chitosan decreased $\mathrm{N}$ intake $(\mathrm{P} \leq 0.05)$, it had no effect on $\mathrm{N}$ milk secretion $(\mathrm{P}>0.05)$ and increased $\mathrm{N}$ efficiency $(\mathrm{P} \leq 0.05)$ in animals fed nonsupplemented diets. Additionally, chitosan and soybean oil had no effect on microbial protein synthesis, allantoin: creatinine ratio, $\mathrm{N}$ urinary excretion and $\mathrm{N}$ balance $(\mathrm{P}>0.05)$.

\subsection{Milk fatty acids profile}

Regardless dietary chitosan addition, soybean oil decreased medium to short chain and increased long chain fatty acids milk concentration ( $P<0.001$; Table 7). A significant soybean oil $\times$ chitosan interaction effect was observed on C16:0 FA, C18:2 cis-9,12 FA, C18:3 c-9,12,15
FA, saturated FA, polyunsaturated FA, n-3 FA and n-6 FA milk concentration $(\mathrm{P}<0.038)$. Chitosan addition in fat supplemented diets had no effect on these milk fatty acids concentration $(P>0.05)$. However, chitosan increased C18:3 cis-9,12,15 FA, C18:2 cis-9,12 FA, poly-unsaturated fatty acids (PUFA), PUFA: saturated ratio, n-3 and n-6 FA concentration and decreased C16:0 FA, and saturated FA concentration when added to diets without soybean oil addition.

\section{Discussion}

Until the present study, there has been no study evaluating chitosan and fat source association in dairy cow diets. Mingoti et al. (2016), Araujo et al. (2015) and Goiri et al. (2010b) found no effect of chitosan on dry matter intake (DMI) in dairy cows, beef cattle and sheep, respectively. In the present study, however, chitosan decreased DMI in

Table 6

Nitrogen and energy usage and microbial protein synthesis of lactating dairy cows fed diets containing chitosan and soybean oil.

\begin{tabular}{|c|c|c|c|c|c|c|c|c|}
\hline \multirow[t]{2}{*}{ Item } & \multicolumn{4}{|l|}{ Diets $^{1}$} & \multirow[t]{2}{*}{ SEM $^{2}$} & \multicolumn{3}{|l|}{ P-value } \\
\hline & $\mathrm{CON}$ & CHI & so & CSO & & Chitosan & Soybean Oil & $\mathrm{CHI} \times \mathrm{SO}^{3}$ \\
\hline \multicolumn{9}{|l|}{ Net energy, MJ/day } \\
\hline Intake (NEI) ${ }^{4}$ & $159^{\mathrm{a}}$ & $148^{\mathrm{b}}$ & $148^{\mathrm{b}}$ & $147^{\mathrm{b}}$ & 2.81 & 0.008 & 0.001 & 0.014 \\
\hline Milk (NEL) ${ }^{5}$ & 96.6 & 96.6 & 87.9 & 86.2 & 1.75 & 0.638 & $<0.001$ & 0.583 \\
\hline \multicolumn{9}{|l|}{ Nitrogen, g/day } \\
\hline Intake & $623^{\mathrm{a}}$ & $590^{\mathrm{b}}$ & $545^{c}$ & $552^{\mathrm{c}}$ & 11.6 & 0.093 & $<0.001$ & 0.009 \\
\hline Urinary & 204 & 206 & 188 & 205 & 6.38 & 0.310 & 0.355 & 0.395 \\
\hline Fecal & 162 & 137 & 133 & 122 & 3.98 & $<0.001$ & $<0.001$ & 0.183 \\
\hline Milk & $158^{\mathrm{a}}$ & $160^{\mathrm{a}}$ & $159^{\mathrm{a}}$ & $150^{\mathrm{b}}$ & 2.82 & 0.236 & 0.102 & 0.047 \\
\hline Balance $^{6}$ & 99.2 & 87.1 & 64.0 & 74.7 & 8.86 & 0.943 & 0.061 & 0.257 \\
\hline Microbial protein & 284 & 299 & 303 & 303 & 10.9 & 0.644 & 0.488 & 0.636 \\
\hline \multicolumn{9}{|l|}{ Coefficients } \\
\hline Energy efficiency $^{7}$ & $0.336^{\mathrm{b}}$ & $0.361^{\mathrm{a}}$ & $0.342^{\mathrm{b}}$ & $0.320^{c}$ & 0.0071 & 0.753 & 0.008 & 0.004 \\
\hline Nitrogen efficiency $^{8}$ & $0.255^{\mathrm{c}}$ & $0.277^{\mathrm{b}}$ & $0.300^{\mathrm{a}}$ & $0.277^{\mathrm{b}}$ & 0.0054 & 0.936 & $<0.001$ & $<0.001$ \\
\hline Allantoin: Creatinine ${ }^{9}$ & 1.52 & 1.57 & 1.68 & 1.60 & 0.053 & 0.857 & 0.274 & 0.446 \\
\hline
\end{tabular}

\footnotetext{
${ }^{1}$ CON: Control diet; CHI: Chitosan diet; SO: Soybean oil diet; and CSO: Chitosan and soybean oil diet.

${ }^{2}$ SEM: Standard error of the mean.

${ }^{3}$ Chitosan $\times$ Soybean oil interaction effect.

${ }^{4}$ NEI: Net energy intake.

${ }^{5}$ Net energy for lactation.

${ }^{6}$ Nitrogen balance (g/day): $\mathrm{N}$ intake - (urinary $\mathrm{N}+$ fecal $\mathrm{N}+$ milk $\mathrm{N}$ ).

${ }^{7}$ Energy efficiency: net energy intake used for lactation (NEL) to digestible energy intake (DEI) ratio.

${ }^{8}$ Nitrogen efficiency: relation between milk nitrogen and nitrogen intake.

${ }^{9}$ Molar ratio.

a,b,c Values within a row with different superscripts differ significantly at $P<0.05$.
} 
Table 7

Milk fatty acids profile of dairy cows fed diets containing chitosan and soybean oil.

\begin{tabular}{|c|c|c|c|c|c|c|c|c|}
\hline \multirow[t]{2}{*}{ Item } & \multicolumn{4}{|l|}{ Diets $^{1}$} & \multirow[t]{2}{*}{$\mathrm{SEM}^{2}$} & \multicolumn{3}{|l|}{ P-value } \\
\hline & $\mathrm{CON}$ & $\mathrm{CHI}$ & so & CSO & & Chitosan & Soybean oil & Int. $\mathrm{Chi} \times \mathrm{SO}^{3}$ \\
\hline \multicolumn{9}{|c|}{ Milk fatty acids concentration, $\mathrm{g} / \mathrm{kg}$} \\
\hline $\mathrm{C} 4: 0$ & 10.6 & 10.7 & 9.0 & 9.0 & 0.22 & 0.860 & $<0.001$ & 0.733 \\
\hline C6:0 & 12.8 & 12.8 & 8.5 & 8.3 & 0.32 & 0.734 & $<0.001$ & 0.741 \\
\hline C8:0 & 9.54 & 9.43 & 5.50 & 5.25 & 0.29 & 0.373 & $<0.001$ & 0.716 \\
\hline C10:0 & 24.5 & 23.9 & 13.4 & 12.7 & 0.81 & 0.323 & $<0.001$ & 0.947 \\
\hline $\mathrm{C} 11: 0$ & 0.64 & 0.62 & 0.35 & 0.32 & 0.03 & 0.523 & $<0.001$ & 0.798 \\
\hline C12:0 & 31.2 & 30.0 & 17.7 & 16.9 & 0.99 & 0.227 & $<0.001$ & 0.798 \\
\hline C13:0 & 2.12 & 2.12 & 1.25 & 1.25 & 0.08 & 0.646 & $<0.001$ & 0.640 \\
\hline C14:0 & 104 & 99.4 & 71.9 & 70.7 & 2.22 & 0.104 & $<0.001$ & 0.351 \\
\hline C14:1 cis-9 & 10.6 & 10.0 & 7.0 & 6.9 & 0.34 & 0.317 & $<0.001$ & 0.541 \\
\hline C15:0 & 10.3 & 10.8 & 7.2 & 7.3 & 0.34 & 0.528 & $<0.001$ & 0.608 \\
\hline C16:0 & $311^{\mathrm{a}}$ & $293^{\mathrm{b}}$ & $235^{\mathrm{c}}$ & $238^{c}$ & 4.48 & 0.039 & $<0.001$ & 0.003 \\
\hline C16:1 cis-9 & 16.8 & 15.8 & 12.5 & 12.7 & 0.46 & 0.464 & $<0.001$ & 0.320 \\
\hline C17:0 & 6.32 & 6.35 & 4.60 & 4.67 & 0.13 & 0.691 & $<0.001$ & 0.883 \\
\hline C18:0 & 95.6 & 99.9 & 141 & 143 & 3.43 & 0.371 & $<0.001$ & 0.715 \\
\hline C18:1 trans-9 & 2.65 & 2.82 & 6.54 & 6.09 & 0.25 & 0.527 & $<0.001$ & 0.159 \\
\hline C18:1 trans-11 & 9.84 & 12.4 & 28.9 & 27.5 & 1.49 & 0.717 & $<0.001$ & 0.243 \\
\hline C18:1 cis-9 & 259 & 268 & 317 & 320 & 4.88 & 0.176 & $<0.001$ & 0.549 \\
\hline C18:2 cis-9,12 & $28.4^{c}$ & $32.2^{\mathrm{b}}$ & $38.0^{\mathrm{a}}$ & $38.3^{\mathrm{a}}$ & 0.80 & 0.012 & 0.319 & 0.031 \\
\hline $\mathrm{C} 18: 2 c-9 t-11$ & 3.92 & 4.74 & 8.02 & 7.54 & 0.34 & 0.726 & $<0.001$ & 0.181 \\
\hline $\mathrm{C} 18: 2 t-10 c-12$ & 0.02 & 0.01 & 0.27 & 0.28 & 0.02 & 0.927 & $<0.001$ & 0.867 \\
\hline $\mathrm{C} 18: 3 c-9,12,15$ & $1.63^{\mathrm{c}}$ & $2.05^{\mathrm{b}}$ & $2.60^{\mathrm{a}}$ & $2.58^{\mathrm{a}}$ & 0.08 & 0.024 & $<0.001$ & 0.015 \\
\hline $\mathrm{C} 20: 0$ & 1.05 & 1.09 & 1.43 & 1.46 & 0.03 & 0.300 & $<0.001$ & 0.978 \\
\hline C20:1 cis-11 & 0.40 & 0.40 & 0.54 & 0.53 & 0.01 & 0.710 & $<0.001$ & 0.845 \\
\hline C20:2 cis-11,14 & 0.06 & 0.09 & 0.05 & 0.09 & 0.02 & 0.279 & 0.809 & 0.809 \\
\hline $\mathrm{C} 20: 3 c-11,14,17$ & $0.95^{\mathrm{b}}$ & $1.10^{\mathrm{a}}$ & $0.84^{c}$ & $0.78^{c}$ & 0.03 & 0.190 & $<0.001$ & 0.003 \\
\hline $\mathrm{C} 20: 4 c-5,8,11,14$ & 1.75 & 1.92 & 1.25 & 1.27 & 0.04 & 0.047 & $<0.001$ & 0.107 \\
\hline $\mathrm{C} 22: 0$ & 0.06 & 0.15 & 0.30 & 0.27 & 0.02 & 0.410 & $<0.001$ & 0.068 \\
\hline Others & 44.1 & 45.5 & 52.3 & 52.8 & 0.83 & 0.492 & 0.859 & 0.738 \\
\hline $\mathrm{SCFA}^{4}$ & 23.4 & 23.5 & 17.5 & 17.3 & 0.51 & 0.944 & $<0.001$ & 0.703 \\
\hline MCFA $^{5}$ & 68.0 & 64.7 & 38.2 & 36.5 & 2.10 & 0.122 & $<0.001$ & 0.622 \\
\hline LCFA $^{6}$ & 864 & 866 & 888 & 892 & 2.42 & 0.268 & $<0.001$ & 0.555 \\
\hline SAT $^{7}$ & $620^{\mathrm{a}}$ & $600^{\mathrm{b}}$ & $517^{\mathrm{c}}$ & $519^{c}$ & 6.85 & 0.098 & $<0.001$ & 0.038 \\
\hline MUFA $^{8}$ & 299 & 309 & 372 & 374 & 5.74 & 0.234 & $<0.001$ & 0.398 \\
\hline PUFA $^{9}$ & $36.8^{\mathrm{c}}$ & $42.1^{\mathrm{b}}$ & $51.5^{\mathrm{a}}$ & $50.9^{\mathrm{a}}$ & 0.97 & 0.013 & $<0.001$ & 0.003 \\
\hline$n-3$ & $1.63^{\mathrm{c}}$ & $2.05^{\mathrm{b}}$ & $2.60^{\mathrm{a}}$ & $2.58^{\mathrm{a}}$ & 0.08 & 0.024 & $<0.001$ & 0.015 \\
\hline$n-6$ & $35.2^{\mathrm{c}}$ & $40.1^{\mathrm{b}}$ & $48.8^{\mathrm{a}}$ & $48.4^{\mathrm{a}}$ & 0.90 & 0.013 & $<0.001$ & 0.003 \\
\hline PUFA: SAT ratio & $0.060^{c}$ & $0.071^{b}$ & $0.100^{\mathrm{a}}$ & $0.099^{\mathrm{a}}$ & 0.026 & 0.057 & $<0.001$ & 0.017 \\
\hline
\end{tabular}

${ }^{1}$ CON: Control diet; CHI: Chitosan diet; SO: Soybean oil diet; and CSO: Chitosan and soybean oil diet.

2 SEM: Standard error of the mean.

${ }^{3}$ Chitosan $\times$ Soybean oil interaction effect.

${ }^{4}$ Short chain fatty acids (C4:0 and C6:0).

${ }^{5}$ Medium chain fatty acids (C8:0 to C13:0 fatty acids).

${ }^{6}$ Long chain fatty acids (C14:0 and higher FA's).

${ }^{7}$ Saturated fatty acids.

${ }^{8}$ mono-unsaturated fatty acids.

${ }^{9}$ poly-unsaturated fatty acids.

a,b,c Values within a row with different superscripts differ significantly at $\mathrm{P}<0.05$.

diets without soybean oil. We expected a chitosan ruminal fermentation modulation effect (Goiri et al., 2010b), similarly to monensin, which could lead to decreased dry matter intake (Duffield et al., 2008). However, chitosan had no effect on ruminal fermentation and, only, increased DM and CP digestibility.

Chitosan increased DM and CP digestibility. Araujo et al. (2015), Paiva et al. (2017), and Mingoti et al. (2016) also found DM and CP digestibility increase using dietary chitosan. These studies and the present one used internal markers for daily fecal estimation. According to Benediktsdottir et al. (2014), in in vitro studies, chitosan can interact with intestinal components, increasing drugs epithelial permeability. These authors did not explain what the possible mechanisms were, and they were not sure whether chitosan acts on transcellular or paracellular processes or both mechanisms. We speculate that chitosan could, similarly, increase ruminant intestinal membranes permeability, increasing nutrients digestibility.

Soybean oil increased propionate concentration and consequently decreased acetate to propionate ratio. Fat effect on rumen fermentation can be attributed to direct action on ruminal microorganisms. Machmuller et al. (1998) found lipids action on protozoa, which are known to be inefficient in dietary energy use. Dohme et al. (2001) showed that lipids inhibited methane production not only by reducing the number of protozoa but also by direct action on other methanogenic populations. Whereby propionate and methane are competing substances for hydrogen reception, lipid addition can increase ruminal propionate concentration and decrease methane synthesis (Eugene et al. (2004)). Likewise, Wencelova et al. (2014) found that chitosan inhibits some protozoan populations, but with lower intensity than vegetables oils. In this study, however, chitosan had no effect on ruminal VFA profile and microbial protein synthesis, using purine derivatives as marker. Water solubility of chitosan is one of the most important characteristic that determines antimicrobial effect (Kong et al., 2010). Furthermore, lower solubility can be associated with small effects on ruminal fermentation and higher effects on intestinal 
digestibility, as observed for Araujo et al. (2015) and Mingoti et al. (2016), using chitosan from Polymar. Fat supplementation can affect intestinal environmental and consequently change chitosan effect on digestion.

Chitosan increased serum urea of cows fed diets without soybean oil. Schelling (1984) suggested that monensin increased the flow of undegraded protein from the rumen to the small intestine because of the decrease in rumen ammonia concentrations likely occurred through the inhibition of deaminated bacteria. Chitosan did not change ruminal ammonia and we agree that serum ammonia increased in response to increased CP digestibility. Increased serum urea concentration indicate higher effect of chitosan on protein digestibility in those animals fed diets without soybean oil. Regardless chitosan addition, soybean oil increased total and HDL serum cholesterol, which can be associated with higher fatty acid intake of these animals. The AST and GGT results with the chitosan addition, regardless of supplementation with soybean oil, showed low hepatotoxicity of this additive, as was also reported by Araujo et al. (2015) and Mingoti et al. (2016).

Chitosan increased feed conversion efficiency in those animals fed diets without soybean oil. This effect seem associated with higher digestibility and lower intake. Paiva et al. (2017) observed milk yield increase and no effect on intake whith chitosan dietary use. However, in fat supplemented diets, chitosan addition decreased milk yield. Mingoti et al. (2016) observed no effect of chitosan on milk yield, using higher ether extract dietary level than Paiva et al. (2016) (42.3 vs $28.9 \mathrm{~g} / \mathrm{kg}$, respectively). One possibility to explain the reduction in feed conversion efficiency is chitosan chelating capacity with many metal ions (Kurita, 1998), which, associated with the changes in the absorptive mechanism of diets supplemented with soybean oil, like calcium salts of fatty acids formation, could determine changes in intermediary metabolism and reduce the efficiency of nutrient utilization.

Soybean oil supplementation, regardless chitosan inclusion, decreased milk fat yield and content. Shingfield et al. (2006) showed that unsaturated fatty acid addition in dairy cow diets decreased milk fat content, which was related to changes in rumen function (Jenkins, 1993). According to Von Soosten et al. (2012), milk fat synthesis might represent up to $50 \%$ of the energy requirement of a high-production dairy cow. Thus, in this study, soybean oil supplementation decreased fat corrected milk yield and energy used for lactation. Moreover, chitosan did not inhibit milk fat depression in diets containing soybean oil.

Soybean oil decreased milk short and medium chain fatty acid concentration and milk fat content. The inhibiting effect of unsaturated C18 on de-novo synthesis has been known in the past, but precise mechanisms involved in the studies were not yet well understood, even if some 18:2 isomers, such as C18:2 trans-10, cis-12, have been identified (Bauman and Griinari, 2001). Renno et al. (2013) also found milk fat depression and short-chain fatty acid concentration decrease in dairy cows fed with long-chain unsaturated fatty acids. In the present study, soybean oil increased milk C18:2 cis-9 trans-11, C18:2 trans-10 cis-12 and decreased short and medium chain fatty acid concentration and chitosan was not able to inhibit linoleic acid biohydrogenation in fat supplemented diets.

Yinghui et al. (2007), studying chitosan cytotoxicity, found specific chemical modifications of chitosan molecule associated with linoleic acid. Association changed molecular charge density and cationic functionalities, structure and conformational flexibility. This may be another reason for absence of effect of chitosan on milk fat profile of those animals fed with fat supplemented diets. Mingoti et al. (2016) also found no effect of chitosan on milk fatty acids profile using lower dietary concentration of ether extract from whole soybeans. In oil-free diets, chitosan increased linoleic and linolenic acid milk concentration. According to Goiri et al. (2010a), chitosan increases vaccenic acid and total conjugated linoleic acid (CLA) in the rumen and this can be associated with chitosan and free fatty acid association and inhibitory effect on the growth of microbial populations.

\section{Conclusion}

Chitosan improved animal performance and nutrient utilization efficiency, increasing long chain fatty acid concentration, showing a very promising feed additive in dairy cow diets without fat supplementation. The addition of chitosan in diets containing soybean oil compromised performance, showing negative interaction of chitosan with basal diet components.

\section{Conflict of interests}

The authors declare that are no conflicts of interest to the current manuscript.

\section{Acknowledgements}

The authors thank the University of São Paulo and Dairy Cattle Research Laboratory for providing the physical infrastructure and staff necessary for this study. We are also grateful for the scholarship support to Tiago Antonio Del Valle from FAPESP (2012/10.420-4) and financial support and grants from Brazilian National Research Council (CNPq).

\section{References}

AOAC, 2000. Official Methods of Analysis of AOAC International, 17th ed. AOAC International, Gaithersburg, MD, USA.

Araújo, A.P.C., Venturelli, B.C., Santos, M.C.B., Gardinal, R., Cônsolo, N.R.B., Calomeni, G.D., Freitas Júnior, J.E., Barletta, R.V., Gandra, J.R., Paiva, P.G., Rennó, F.P., 2015 Chitosan affects total nutrient digestion and ruminal fermentation in Nellore steers. Anim. Feed Sci. Technol. 206, 114-118. http://dx.doi.org/10.1016/j.anifeedsci. 2015.05.016.

Arndt, C., Powell, J.M., Aguerre, M.J., Crump, P.M., Wattiaux, M.A., 2015. Feed conversion efficiency in dairy cows: repeatability, variation in digestion and metabolism of energy and nitrogen, and ruminal methanogens. J. Dairy Sci. 98, 3938-3950.

Barton, M.D., 2000. Antibiotic use in animal feed and its impact on human healt. Nutr. Res. Rev. 13 (279-279).

Bauman, D.E., Griinari, J.M., 2001. Regulation and nutritional manipulation of milk fat: low fat milk syndrome. Livest. Prod. Sci. 70, 15-29.

Benediktsdottir, B.E., Baldursson, O., Masson, M., 2014. Challenges in evaluation of chitosan and trimethylated chitosan (TMC) as mucosal permeation enhancers: from synthesis to in vitro application. J. Control. Release 173, 18-31.

Broderick, G.A., Kang, J.H., 1980. Automated simultaneous determination of ammonia and total amino-acids in ruminal fluid and in vitro media. J. Dairy Sci. 63, 64-75.

Casali, A.O., Detmann, E., Valadares Filho, S.C., Pereira, J.C., Henriques, L.T., Freitas, S.G., Paulino, M.F., 2008. Influence of incubation time and particles size on indigestible compounds contents in cattle feeds and feces obtained by in situ procedures. Braz. J. Anim. Sci. 37, 335-342.

Chen, X.B., Gomes, M.J., 1992. Estimation of Microbial Protein Supply to Sheep and Cattle Based on Urinary Excretion of Purine Derivatives - An Overview of the Technical Details. Ocasional publication 1992, International Feed Resources Unit, Rowett Research Institute, Aberdeen, UK., pp. 22.

Chizzotti, M.L., Valadares Filho, S.C., Valadares, R.F.D., Chizzotti, F.H.M., Tedeschi, L.O., 2008. Determination of creatinine excretion and evaluation of spot urine sampling in Holstein cattle. Livest. Prod. Sci. 113, 218-225.

Dohme, F., Machmuller, A., Wasserfallen, A., Kreuzer, M., 2001. Ruminal methanogenesis as influenced by individual fatty acids supplemented to complete ruminant diets. Lett. Appl. Microbiol. 32, 47-51.

Duffield, T.F., Rabiee, A.R., Lean, A.J., 2008. A meta-analysis of the impact of monensin in lactating dairy cattle. Part 2. Production effects. J. Dairy Sci. 91, 1347-1360.

Eugene, M., Archimede, H., Sauvant, D., 2004. Quantitative meta-analysis on the effects of defaunation of the rumen on growth, intake and digestion in ruminants. Livest. Prod. Sci. 85, 81-97.

Feng, S., Lock, A.L., Garnsworthy, P.C., 2004. Technical note: a rapid lipid separation method for determining fatty acid composition of milk. J. Dairy Sci. 87, 3785-3788.

Fujihara, S., Yamaguchi, M., 1978. Effects of allopurinol 4-hydroxypyrazolo(3,4-d) pyrimidine on metabolism of allantoin in soybean plants. Plant Physiol. 62, 134-138.

Goiri, I., Oregui, L.M., Garcia-Rodriguez, A., 2009. Dose-response effects of chitosans on in vitro rumen digestion and fermentation of mixtures differing in forage-toconcentrate ratios. Anim. Feed Sci. Technol. 151, 215-227.

Goiri, I., Indurain, G., Insausti, K., Sarries, V., Garcia-Rodriguez, A., 2010a. Ruminal biohydrogenation of unsaturated fatty acids in vitro as affected by chitosan. Anim. Feed Sci. Technol. 159, 35-40.

Goiri, I., Oregui, L.M., Garcia-Rodriguez, A., 2010b. Use of chitosans to modulate ruminal fermentation of a 50:50 forage-to-concentrate diet in sheep. J. Anim. Sci. 88, 749-755.

González-Ronquillo, M., Balcells, J., Guada, J.A., Vicente, F., 2003. Purine derivative excretion in dairy cows: endogenous excretion and the effect of exogenous nucleic 
acid supply. J. Dairy Sci. 86, 1282-1291.

Hall, M.B., 2000. Calculation of non-structural carbohydrate content of feeds that contain non-protein nitrogen. Univ. Fla. P. A 25 (Bulletin 339).

Jenkins, T.C., 1993. Lipid-metabolism in the rumen. J. Dairy Sci. 76, 3851-3863.

Kenward, M., Roger, J., 1997. Small Sample Inference for Fixed Effects from Restricted Maximum Likelihood. Biometrics 53, 983-997.

Kong, M., Chen, X.G., Xing, K., Park, H.J., 2010. Antimicrobial properties of chitosan and mode of action: a state of the art review. Int J. Food Microbiol. 144, 51-63.

Kramer, J.K.C., Fellner, V., Dugan, M.E.R., Sauer, F.D., Mossoba, M.M., Yurawecz, M.P., 1997. Evaluating acid and base catalysts in the methylation of milk and rumen fatty acids with special emphasis on conjugated dienes and total trans fatty acids. Lipids 32, 1219-1228.

Kurita, K., 1998. Chemistry and application of chitin and chitosan. Polym. Degrad. Stab. 59, 117-120.

Loften, J.R., Linn, J.G., Drackley, J.K., Jenkins, T.C., Soderholm, C.G., Kertz, A.F., 2014. Invited review: palmitic and stearic acid metabolism in lactating dairy cows. J. Dairy Sci. 97, 4661-4674.

Machmuller, A., Ossowski, D.A., Wanner, M., Kreuzer, M., 1998. Potential of various fatty feeds to reduce methane release from rumen fermentation in vitro (Rusitec). Anim. Feed Sci. Tech. 71, 117-130. http://dx.doi.org/10.1016/S0377-8401(97)00126-0.

Mingoti, R.D., Freitas, Jr., J.E., Gandra, J.R., Gardinal, R., Calomeni, G.D., Barletta, R.V., Vendramini, T.H.A., Paiva, P.G., Rennó, F.P., 2016. Dose response of chitosan on nutrient digestibility, blood metabolites and lactation performance in holstein dairy cows. Livest. Sci. 187, 35-39. http://dx.doi.org/10.1016/j.livsci.2016.02.008.

National Research Council (NRC), 2001. Nutrient Requirements of Dairy Cattle, Seventh revised. National Academy Press, Washington, DC, USA (381p).

Odongo, N.E., Bagg, R., Vessie, G., Dick, P., Or-Rashid, M.M., Hook, S.E., Gray, J.T., Kebreab, E., France, J., McBride, B.W., 2007. Long-term effects of feeding monensin on methane production in lactating dairy cows. J. Dairy Sci. 90, 1781-1788.

Paiva, P.G., Ferreira de Jesus, E., Del Valle, T.A., Almeida, G.F., Costa, A.G.B.V.B., Consentini, C.E.C., Zanferari, F., Takiya, C.S., Bueno, I.C.S., Rennó, F.P., 2017. Effects of chitosan on ruminal fermentation, nutrient digestibility, and milk yield and composition of dairy cows. Anim. Prod. Sci. 57, 301-307. http://dx.doi.org/10. 1071/AN15329.

Renno, F.P., Freitas Junior, J.E., Gandra, J.R., Verdurico, L.C., Santos, M.V., Venturelli, Barletta, R.V., B.C. Vilela, F.G., 2013. Fatty acid profile and composition of milk protein fraction in dairy cows fed long-chain unsaturated fatty acids during the transition period. Braz. J. Anim. Sci. 42, 813-823.

SAS Institute, Inc, 2003. SAS User's Guide: Statistics, Version 9.0. SAS Institute, Inc., Cary, NC, USA.

Schelling, G.T., 1984. Monensin mode of action in the rumen. J. Anim. Sci. 58, 1518-1526.

Shen, Z.M., Seyfert, H.M., Lohrke, B., Schneider, F., Zitnan, R., Chudy, A., Kuhla, S., Hammon, H.M., Blum, J.W., Martens, H., Hagemeister, H., Voigt, J., 2004. An energy-rich diet causes rumen papillae proliferation associated with more IGF type 1 receptors and increased plasma IGF-1 concentrations in young goats. J. Nutr. 134, 11-17.

Shingfield, K.J., Reynolds, C.K., Hervas, G., Griinari, J.M., Grandison, A.S., Beever, D.E., 2006. Examination of the persistency of milk fatty acid composition responses to fish oil and sunflower oil in the diet of dairy cows. J. Dairy Sci. 89, 714-732.

Silva, D.C., Santos, G.T., Branco, A.F., Damasceno, J.C., Kazama, R., Matsushita, M., Horst, J.A., Santos, V., Petit, H.V., 2007. Production performance and milk composition of dairy cows fed whole or ground flaxseed with or without monensin. J. Dairy Sci. 90, 2928-2936.

Sklan, D., Kaim, M., Moallem, U., Folman, Y., 1994. Effect of dietary calcium soaps on milk-yield, bidy-weight, reproductive hormones and fertility in 1st parity and older cows. J. Dairy Sci. 77, 1652-1660.

Van Nevel, C.J., Demeyer, D.I., 1988. Manipulation of Rumen Fermentation. The Rumen Microbial Ecosystem. Elsevier Science Publishing, New York (NY387-443).

Van Soest, P.J., Robertson, J.B., Lewis, B.A., 1991. Methods for dietary fiber, neutral detergent fiber, and nonstarch polysaccharides in relation to animal nutrition. J. Dairy Sci. 74, 3583-3597.

Von Soosten, D., Meyer, U., Piechotta, M., Flachowsky, G., Daenicke, S., 2012. Effect of conjugated linoleic acid supplementation on body composition, body fat mobilization, protein accretion, and energy utilization in early lactation dairy cows. J. Dairy Sci. 95, 1222-1239.

Wencelova, M., Varadyova, Z., Mihalikova, K., Kisidayova, S., Jalc, D., 2014. Evaluating the effects of chitosan, plant oils, and different diets on rumen metabolism and protozoan population in sheep. Turk. J. Vet. Anim. Sci. 38, 26-33.

Yinghui, L.V., Chen, X., Wang, Q., Wang, Y., Zhang, J., Liu, C., Liu, C., Meng, X., Yu, L., 2007. Synthesis and characterization of chitosan-based biomaterials modified with different active groups and their relationship with cytotoxicity. J. Wuhan Univ. Technol. 22, 695-700. 